\title{
THE CONVERGENCE OF THE GALERKIN METHOD FOR THE TAYLOR-DEAN STABILITY PROBLEM*
}

\author{
By R. C. DIPRIMA and R. SANI $\dagger$ (Rensselaer Polytechnic Institute, Troy, New York)
}

1. Introduction. In many investigations in hydrodynamic and hydromagnetic stability the Galerkin method has been used to solve the resulting eigenvalue problem. Typical problems are the stability of flow between rotating concentric cylinders (the Taylor problem); the stability of flow between concentric cylindrical surfaces with a circumferential pressure gradient (the Dean problem); the stability of the combined flows (the Taylor-Dean problem); and the stability of the above-mentioned flows with axial or circumferential magnetic fields. The formulation of such stability problems usually leads to a linear non-selfadjoint eigenvalue problem involving a sixth-order (or higher) differential equation.

Thus, for example, in the case that the gap between the cylinders is small compared to the mean radius, the Taylor-Dean stability problem leads to the sixth-order system

$$
\begin{gathered}
\left(D^{2}-a^{2}\right)^{2} u=f(x) v, \\
\left(D^{2}-a^{2}\right) v=-a^{2} T g(x) u,
\end{gathered}
$$

on $0 \leq x \leq 1$ with the boundary conditions

$$
u=v=D u=0
$$

at $x=0$ and $x=1$. (See Chandrasekhar [1, Sec. 76] or DiPrima [2] for a derivation of these equations.) Here $D$ denotes differentiation with respect to $x, a$ is the dimensionless wave number of the disturbance, $f(x)$ and $g(x)$ are known continuous functions, and $T$ is a parameter depending upon a characteristic velocity of the system. In general $f(x)$ and $g(x)$ will not be of one sign on $0 \leq x \leq 1$. In particular, the classical Taylor stability problem is of the form (1), (2), and (3) with $g(x)=1$ and $f(x)=1-(1-\mu) x$, $\mu<1$. The mathematical problem is, for given $f(x)$ and $g(x)$, to determine the minimum real positive $T$ over all real positive $a$ for which there exists a solution of the system (1), (2) and (3).

In using the Galerkin method to solve the resulting eigenvalue problem, the eigenfunction is expanded in a complete (in an appropriate norm) set of functions satisfying the boundary conditions:

$$
u(x)=\sum_{i=1}^{\infty} A_{i} u_{i}(x), \quad v(x)=\sum_{i=1}^{\infty} B_{i} v_{i}(x)
$$

with $u_{i}=D u_{i}=v_{i}=0$ at $x=0$ and $x=1$. These series are then introduced into the differential equations (1) and (2). The requirement that the error in the differential equations (1) and (2) be orthogonal to the $u_{i}$ and the $v_{i}$ respectively leads to a system of infinitely many linear homogeneous equations for the $A_{i}$ and $B_{i}$. The necessity of

*Received July 6, 1964; revised manuscript received December 2, 1964. This work was supported by the Mechanics Branch of the Office of Naval Research, Contract Nonr-591(08) and by the National Science Foundation, Grant No. GP-2337.

$\dagger$ Present address, Department of Chemical Engineering, University of Illinois, Urbana, Illinois. 
the vanishing of the determinant associated with this system of equations gives the desired eigenvalue equation. Approximate values of the eigenvalues are obtained by truncating the series for $u$ and $v$ after a finite number of terms. If the eigenvalue problem admits of a formulation as a variational principle or a minimum principle, it is known that the Galerkin method is equivalent to such a formulation, and under suitable conditions on the expansion functions the convergence of the approximate eigenvalues to the exact eigenvalues as more terms are taken in the series can be proved.

It is the purpose of this note to show that for the non-selfadjoint eigenvalue problem (1), (2), and (3), the approximate eigenvalues as determined by the Galerkin method converge to the exact eigenvalues as the number of terms in the series for $u$ and $v$ are increased. The proof is based on the following theorem by Mikhlin [3, Sec. 52].

Theorem. Let $\lambda$ be a parameter in the equation

$$
A w-\lambda K w=0,
$$

where $A$ and $K$ are linear operators, and the domain of $A, D_{A}$, is a linear manifold, that is dense in a Hilbert space $H$ with inner product $($,$) . Let D_{A}$ be contained in the domain of $K$, and assume that the following conditions are fulfilled:

1. The operator $A$ is a positive-definite, self-adjoint operator; that is, $(A u, u)>0$ and $(A u, v)-(v, A u)=0$.

2. The operator $A^{-1} K$ can be extended to be completely continuous on the Hilbert space $H_{0}$ which is the completion of $D_{A}$ under the norm $(A u, u)^{1 / 2}$.

Then the Galerkin method for calculating the eigenvalues of (5) is a convergent process in $H_{0}$.

In the following section we will discuss the nomenclature of this theorem and will show that the eigenvalue problem (1), (2), and (3) can be cast in a form such that the conditions of the theorem are satisfied. The extension to a number of other hydrodynamic and hydromagnetic stability problems is straightforward.

The convergence of the Galerkin method for the Taylor stability problem, $g \equiv 1$ in Eq. (2), has also been considered by Kolomy [4]. He considered the sixth-order equation $\left(D^{2}-a^{2}\right)^{3} v=-a^{2} T f(x) v$ which is obtained by eliminating $u$ between Eqs. (1) and (2). His proof that the Galerkin method converges for this sixth-order equation appears, however, to be incorrect. In the proof, it is assumed that the sixth-order operator $\left(D^{2}-a^{2}\right)^{3}$ is symmetric in the sense of an $L_{2}$ inner product on a space of functions satisfying $v=D^{2} v=D\left(D^{2}-a^{2}\right) v=0$ at $x=0$ and $x=1$. However, it can easily be established by direct integration by parts that this is not true. In fact, it has been shown by Sani [5] that it is necessary to use a generalized Galerkin method to treat the sixth-order equation.

2. The convergence proof. The differential equations (1), (2) can be put in the form (4) by letting $\lambda=a T^{1 / 2}$, and rescaling $u$ by a factor $1 / \lambda$. Then we have

$$
A \mathrm{w}=\lambda K \mathrm{w},
$$

where $\mathrm{w}(x)$ is the column vector with components $u$ and $v$, and $A$ and $K$ are the linear matrix operators

$$
A=\left[\begin{array}{cc}
\left(D^{2}-a^{2}\right)^{2} & 0 \\
0 & -\left(D^{2}-a^{2}\right)
\end{array}\right], K=\left[\begin{array}{cc}
0 & f(x) \\
g(x) & 0
\end{array}\right]
$$


The domain, $D_{A}$, of the linear operator $A$ is the space of two dimensional complexvalued vectors $\mathrm{w}(x)$ whose first component has continuous derivatives through the fourth order on $0<x<1$ and satisfies the boundary conditions $u=D u=0$ at $x=0$ and $x=1$, and whose second component has continuous derivatives through the second order and satisfies the boundary conditions $v=0$ at $x=0$ and $x=1$. This set is dense in the Hilbert space $L_{2}$ of square integrable complex-valued two dimensional vector functions on $0 \leq x \leq 1$, with inner product*

$$
\left(\mathbf{w}_{1}, \mathbf{w}_{2}\right)=\int_{0}^{1}\left(u_{1} u_{2}^{c}+v_{1} v_{2}^{c}\right) d x,
$$

and norm

$$
\|\mathbf{w}\|=(\mathbf{w}, \mathbf{w})^{1 / 2} .
$$

That is, any function in $L_{2}$ can be approximated arbitrarily closely in the norm given by Eq. (9) by functions in $D_{A}$. See Sec. 3 for examples of sets of functions in $D_{A}$ that are dense in $L_{2}$.

On the space $D_{A}$ it can be readily established by direct integration by parts that $A$ is a positive-definite, self-adjoint operator. For example,

$$
\begin{aligned}
(A \mathrm{w}, \mathbf{w}) & =\int_{0}^{1}\left\{u^{c}\left(D^{2}-a^{2}\right)^{2} u-v^{c}\left(D^{2}-a^{2}\right) v\right\} d x \\
& =\int_{0}^{1}\left\{\left|\left(D^{2}-a^{2}\right) u\right|^{2}+|D v|^{2}+a^{2}|v|^{2}\right\} d x \geq 0, \quad\left(a^{2}>0\right)
\end{aligned}
$$

and the equality holds only for $\mathrm{w}=0$. Similarly,

$$
\left(A \mathbf{w}_{1}, \mathbf{w}_{2}\right)-\left(\mathbf{w}_{1}, A \mathbf{w}_{2}\right)=0 .
$$

Hence condition 1 of the theorem is satisfied.

Clearly, $D_{A}$ is a linear vector space, and is also contained in the domain of $K$ since $K$ is a bounded operator. An inner product on $D_{A}$ can be defined by $\left[\mathrm{w}_{1}, \mathrm{w}_{2}\right]=\left(A \mathrm{w}_{1}, \mathrm{w}_{2}\right)$ with the corresponding norm

$$
\left\||\mathbf{w} \||^{2}=[\mathbf{w}, \mathbf{w}]=\int_{0}^{1}\left\{\left|\left(D^{2}-a^{2}\right) u\right|^{2}+|D v|^{2}+a^{2}|v|^{2}\right\} d x .\right.
$$

With this norm $D_{A}$ is a pre-Hilbert space; that is, all of the conditions of a Hilbert space except completeness are satisfied. The space $D_{A}$ can be completed by adding to $D_{A}$ the limit points of all Cauchy sequences in $D_{A}$ which converge in the sense of the above norm. By construction this space is a Hilbert space, which we call $H_{0}$.

Let $T \equiv A^{-1} K$, then

$$
T \mathbf{w}=\int_{0}^{1} G(x, \xi) K(\xi) \mathbf{w}(\xi) d \xi
$$

where $G$ is the matrix Green's function corresponding to the matrix differential operator $A$ with the boundary conditions (3). Since $K$ and $G$ are uniformly bounded, $T$ is defined on $L_{2}$. By a slight extension of the argument given by Epstein [6, pp. 78-79]

*The superscript $C$ denotes the complex conjugate. 
it follows that $T$ is completely continuous in $L_{2}$. That is, $T$ takes bounded sets in $L_{2}$ into compact sets in $L_{2}$, or equivalently, bounded sequences are taken into sequences with a convergent subsequence. Next we want to show that $T$ is defined and completely continuous on $H_{0}$.

First, according to the Poincaré inequality there exists a real positive constant $\alpha$ such that

$$
\alpha \int_{0}^{1}|\mathbf{w}|^{2} d x \leq \int_{0}^{1}|D \mathbf{w}|^{2} d x .
$$

By repeated application of this inequality it can be shown that there exists a real positive $\beta$ such that

Consequently for w in $H_{0}$,

$$
\beta\|\mathbf{w}\| \leq\|\mathbf{w}\| \text {. }
$$

$$
\begin{aligned}
\left\||T \mathbf{w} \||^{2}\right. & =\left(A A^{-1} K \mathbf{w}, A^{-1} K \mathbf{w}\right) \\
& \leq\|K \mathbf{w}\| \cdot\left\|A^{-1} K \mathbf{w}\right\| \\
& \leq\left.\frac{1}{\beta^{2}}\|K\| \cdot\left\|A^{-1} K\right\| \cdot\|\mathbf{w}\|\right|^{2},
\end{aligned}
$$

where $\|K\|$ and $\left\|A^{-1} K\right\|$ are the norms of $K$ and $A^{-1} K$ on $L_{2}$. Hence, $T$ is defined on $H_{0}$. Finally, consider a bounded set $S$ of functions $\mathbf{w}(x)$ in $H_{0}$, say $\left\||\mathbf{w} \||<B_{0}\right.$. We want to show that there exists a sequence $\left\{\mathbf{w}_{n}\right\}$ in $S$ such that $\left\|\left|T \mathbf{w}_{n}-T \mathbf{w}_{m} \|\right| \rightarrow 0\right.$ as $n, m \rightarrow \infty$. From Eq. (15) it follows that if $\mathrm{w}$ is in $S$, then $\|\mathbf{w}\| \leq B_{0} / \beta$, so that the set $S$ is a bounded set in $L_{2}$. Since $T$ is completely continuous in $L_{2}$, there exists a sequence $\left\{\mathbf{w}_{n}\right\}$ in $S$ such that $\left\|T \mathbf{w}_{n}-T \mathbf{w}_{m}\right\| \rightarrow 0$ as $n, m \rightarrow \infty$. For this sequence

$$
\begin{aligned}
\left\|\mid T \mathbf{w}_{n}-T \mathbf{w}_{m}\right\| \| & =\left(A\left(T \mathbf{w}_{n}-T \mathbf{w}_{m}\right), T \mathbf{w}_{n}-T \mathbf{w}_{m}\right) \\
& =\left(K\left(\mathbf{w}_{n}-\mathbf{w}_{m}\right), T\left(\mathbf{w}_{n}-\mathbf{w}_{m}\right)\right) \\
& \leq\|K\| \cdot\left\|\mathbf{w}_{n}-\mathbf{w}_{m}\right\| \cdot\left\|T \mathbf{w}_{n}-T \mathbf{w}_{m}\right\| .
\end{aligned}
$$

But the right-hand side of Eq. (17) approaches zero as $n, m \rightarrow \infty$; hence, $T \equiv A^{-1} K$ is completely continuous on $H_{0}$ and the conditions of Mikhlin's theorem are established. Thus the Galerkin method for computing the eigenvalues of (6) converges in the norm for $H_{0}$.

3. Remarks. In using the Galerkin method for the eigenvalue problem (6) it is necessary that the expansion functions $\left\{\mathbf{w}_{n}(x)\right\}$ be complete in the norm $\||\mathbf{w}|||$. That is, given an element $\mathrm{w}$ in $H_{0}$, then there must exist $\alpha_{n}$ such that

$$
\left\|\mathbf{w}-\sum_{n=1}^{M} \alpha_{n} \mathbf{w}_{n}\right\| \| 0
$$

as $M \rightarrow \infty$. One suitable set of functions are the polynomials $u_{n}(x)=(1-x)^{2} x^{n+1}$, $n=1,2, \cdots$ for $u(x)$ and the polynomials $v_{n}(x)=(1-x) x^{n}, n=1,2, \cdots$ for $v(x)$. It follows from the Weirstrass approximation theorem that if $u(x)$ has continuous first and second derivatives and vanishes with its first derivative at $x=0$ and $x=1$, then given any $\epsilon>0$ there exists a linear combination of the $u_{n}(x)$ such that

$$
\left|u-\sum_{1}^{M} a_{n} u_{n}\right|<\epsilon, \quad\left|u^{\prime}-\sum_{1}^{M} a_{n} u_{n}^{\prime}\right|<\epsilon, \quad\left|u^{\prime \prime}-\sum_{1}^{M} a_{n} u_{n}^{\prime \prime}\right|<\epsilon,
$$


where primes denote differentiation with respect to $x$. See, for example, Kantorovich and Krylov [7, p. 265]. A similar remark is true for $v(x)$ and the $v_{n}(x)$. Hence the solution of the eigenvalue problem (6) can be approximated arbitrarily closely in the norm $\||\mathbf{w}| \mid$ by these polynomial functions. Alternatively on the interval $-\frac{1}{2} \leq x \leq \frac{1}{2}$ one could use the trigonometric functions $\sin 2 n \pi x$ and $\cos (2 n-1) \pi x$ for $v(x)$ and the functions $C_{n}(x)$ and $S_{n}(x)$ which have been tabulated by Reid and Harris [8] for $u(x)$. Here the trigonometric functions are solutions of the Sturm-Liouville problem $y^{\prime \prime}=-\lambda^{2} y$, $y\left(-\frac{1}{2}\right)=y\left(\frac{1}{2}\right)=0$ and the $C_{n}(x)$ and $S_{n}(x)$ are the even and odd solutions of the SturmLiouville problem $y^{\prime \prime \prime \prime}=\lambda^{4} y, y\left(-\frac{1}{2}\right)=y^{\prime}\left(-\frac{1}{2}\right)=y\left(\frac{1}{2}\right)==y^{\prime}\left(\frac{1}{2}\right)=0$. In a space of functions satisfying the indicated boundary conditions, the former functions are complete in a first derivative norm and the latter functions are complete in a second derivative norm. From this it again follows that the solution of the eigenvalue problem can be approximated arbitrarily closely in the norm $\|\mid \mathbf{w}\|$.

The preceding arguments can be extended in a straightforward manner to show that the Galerkin method is a convergent procedure for computing the eigenvalues of a number of different hydrodynamic and hydromagnetic stability problems. Some of these are the finite-gap Taylor, Dean, and Taylor-Dean problems, and the finite-gap or small-gap Taylor, Dean, or Taylor-Dean problems with an axial magnetic field or with a circumferential magnetic field. The latter problems lead to eigenvalue problems involving 8 th order systems of differential equations but this does not cause any difficulty.

Two additional questions are of interest. The first, which was mentioned earlier, is concerned with the use of the Galerkin method to solve the eigenvalue problem for the classical Taylor problem when it is formulated as a 6th-order differential equation. The second is concerned with the method of Pellew and Southwell [9] which has been modified and used so successfully by Chandrasekhar (see [1]) and others. For this approximate method the idea for the eigenvalue problem (1), (2), and (3) is to expand $v(x)$ in a complete set of functions satisfying the appropriate boundary conditions, then solve Eq. (1) for $u(x)$. A determinantal equation for $T$ is then obtained by requiring that the error in Eq.(2) be orthogonal to the expansion functions for $v(x)$. The convergence of this procedure as well as the first question have been discussed by Sani [5].

\section{ReFERENCES}

1. S. Chandrasekhar, Hydrodynamic and hydromagnetic stability, Oxford University Press, England, 1961

2. R. C. DiPrima, The stability of viscous flow between rotating concentric cylinders with a pressure gradient acting round the cylinders, Journal of Fluid Mechanics, 6, 462-468 (1959)

3. S. Mikhlin, Priamye metody $v$ matematicheskoi fizike, (Direct methods of mathematical physics), GTTI, Moscow, 1950

4. J. Kolomý, Už̃tí Galerkinovy metody v ulohach stabilitě proudĕní vazké tekutiny, (T'he use of the Galerkin method for the problem of the stability of viscous flow), Aplikace Matematiky, 5, 40-44 (1960)

5. R. Sani, Convergence of a generalized Galerkin method for certain fluid stability problems, Renssalaer Polytechnic Institute Math Rep 63, Troy, New York, 1964

6. B. Epstein, Partial differential equations, McGraw-Hill Book Co., New York, 1962

7. L. V. Kantorovich and V. I. Krylov, Approximate methods of higher analysis, P. Noordhoff Ltd., Amsterdam, 1958

8. D. L. Harris and W. H. Reid, On orthogonal functions which satisfy four boundary conditions I. Tables for use in Fourier-type expansions, Astrophysics Journal Supplement, ser. 3, 429-447 (1958)

9. A. Pellew and R. V. Southwell, On maintained convective motion in a fluid heated from below, Proc. Roy. Soc., ser. A, 176, 312-343 (1940) 